\title{
Patterns of Valvular Involvement in Rheumatic Heart Disease patients taking Benzathine Penicillin at Shahid Gangalal National Heart Centre, Kathmandu, Nepal
}

\author{
Rabi Malla, ${ }^{1}$ Suman Thapaliya, ${ }^{1}$ Prakash Gurung, ${ }^{1}$ Amrit Bogati, ${ }^{1}$ Sunita Khadka, ${ }^{2}$ Sajana Shrestha, ${ }^{2}$ \\ Sujeeb Rajbhandari, ${ }^{1}$ Chandra Mani Adhikari. ${ }^{1}$
}

\author{
${ }^{1}$ Department of Cardiology, Shahid Gangalal National Heart Centre, Bansbari, Kathmandu, Nepal. \\ ${ }^{2}$ Department of Nursing, Shahid Gangalal National Heart Centre, Bansbari, Kathmandu, Nepal. \\ Corresponding Author: Chandra Mani Adhikari, Shahid Gangalal National Heart Centre, Bansbari, Kathmandu, Nepal, \\ Email: topjhap@hotmail.com
}

Abstract

\begin{abstract}
Background and Aims: Acute rheumatic fever and rheumatic heart disease constitute an important public health problem in the developing countries. The disease results from an abnormal autoimmune response to a group A streptococcal infection in a susceptible host. We aim to describe the pattern of valvular involvement in patients taking Injection Benzathine Penicillin.

Methods: All the patients, who were taking injection Benzathine penicillin during 15th April to 14th July, 2013 at our Centre, were included in this study. Demographic features like age, sex, echocardiographic diagnosis along with any adverse effects of Benzathine penicillin were collected.

Results: A total of 661 patients were included in our study, out of which female predominated in numbers. Rheumatic heart disease rather than rheumatic fever was the cause for Penicillin injection. Mitral valve was the most common valve involved and it was more common in female.Pure mitral stenosis was the most common valvular involvement. Forty two percent patients underwent intervention; among them Percutaneous transluminal mitral commisuorotomy was the most common. There was no adverse event during the study time following penicillin injection.

Conclusions: Women are more commonly affected than male. Mitral valve is the most common valve involved.
\end{abstract}

Keywords: Benzathine Penicillin, Rheumatic fever, Rheumatic Heart Disease, Valvular involvement

\section{Introduction}

Rheumatic fever (RF) and rheumatic heart disease (RHD) constitute important public health problem in the developing countries due to its high prevalence. RHD is a significant cause of morbidity and mortality among Nepalese school children. ${ }^{1,2}$ Its prevalence is high both in rural as well as in urban Nepal. ${ }^{3}$

The disease results from an abnormal autoimmune response to a group A streptococcal infection in a susceptible host. RF, the precursor to RHD, can affect different organs and lead to irreversible valve damage and heart failure. ${ }^{4}$ RHD occurs in $30-45 \%$ of the patients with RF and it leads to chronic valvular lesions. ${ }^{5}$ Injection Benzathine Penicillin is recommended for the primary and secondary prevention of the disease.

Our study was designed to describe the pattern of valvular involvement in patients taking Benzathine Peniciliin.

\section{Methods}

It was a single centre prospective, cross sectional, descreptive study conducted for three months from 15th April to 14th July, 2013 in Shahid Gangalal National Heart centre (SGNHC), Kathmandu, Nepal. The study protocol was approved by the Ethics Committee of SGNHC. Informed consent was taken from all the patients. All the patients, who were prescribed with injection
Benzathine penicillin during the study period were included in this study. Demographic features like age, sex, echocardiographic findings (valvular involvement) along with any adverse effects of Benzathine penicillin were collected. Echocardiographic diagnosis of RHD was done according to World Heart Federation criteria for echocardiographic diagnosis of RHDan evidence-based guideline. ${ }^{6}$ Statistical analysis was performed with SPSS 16.

\section{Results}

One thousand six hundred and eighty six Benzathine Penicillin injections were given to 661 patients. Age ranged from 4 years to 50 years, with the mean age $24.1 \pm 7.1$ years. Out of 661 patients $391(59.1 \%)$ were female and $270(40.9 \%)$ were male with female to male ratio of $1.44: 1 . \mathrm{RF}$ was the cause for penicillin injection in $78(11.6 \%)$ patients. Five hundred and eighty three $(88.4 \%)$ patients had valvular involvement. The number of female patients was much more than male taking Benzathine penicillin in RF and RHD patients as shown in table 1 . 
Table 1. Difference in frequency of RF and RHD among Male and female

$\begin{array}{lll}\mathbf{R F} & \mathbf{7 8} & \mathbf{1 1 . 6 \%} \\ \text { Male } & 36 & 46.7 \% \\ \text { Female } & 42 & 53.3 \% \\ \text { RHD } & \mathbf{5 8 3} & \mathbf{8 8 . 4 \%} \\ \text { Male } & 234 & 40.1 \% \\ \text { Female } & 349 & 59.9 \%\end{array}$

Solitary valvular involvement of either mitral or aortic valve was present in 491 patients. Isolated mitral valve involvement (Mitral Stenosis (MS), Mitral regurgitation (MR) and MS with MR) was present in 448 patients whereas isolated Aortic valve involvement (Aortic stenosis (AS), Aortic regurgitation (AR) and AR with AS) was present in 43 patients. Lone MS (209 patients) is the most common valvular involvement followed by, Lone MR (201 patients), MS with MR in 38 patients and AR in 33 patients as shown in Table 2.
Mixed (Mitral as well as Aortic) valve involvement was present in 89 patients. Among mixed valvular involvement, MR with $\mathrm{AR}$ was the most common (59 patients) followed by AR with MS (14) and AS with MR in 10 patients. All types of valvular involvement (AR/AS/MR/MS) were a rare combination present in 2 patients as shown in table 2 .

Table 2. Valvular Involvement $\mathrm{n}=583$

\begin{tabular}{lll}
\hline Valve & No & $\%$ \\
\hline Mitral valve & 448 & 76.8 \\
MS & 209 & 35.8 \\
MR & 201 & 34.5 \\
MS with MR & 38 & 6.5 \\
\hline Aortic Value & 43 & 7.4 \\
AS & 5 & 0.8 \\
AR & 33 & 5.7 \\
AS with AR & 5 & 0.8 \\
Mixed involvement & 92 & 15.8 \\
AR with MR & 63 & 10.1 \\
AR with MS & 13 & 2.4 \\
AS with MR & 7 & 1.7 \\
AS with MS & 3 & 0.5 \\
AR/AS/MR/MS & 2 & 0.3 \\
AR/MS/MR & 4 & 0.6 \\
\hline
\end{tabular}

Mitral valve disease was significantly more in female compared to male whereas Aortic valve involvement was significantly more common in Male patients.

Table 3. Male female ratios among valve lesion

\begin{tabular}{lllll}
\hline Valve & Total & Male & Female & Male/Female ratio \\
\hline Mitral valve & 448 & 161 & 287 & 0.56 \\
MS & 209 & 71 & 138 & 0.51 \\
MR & 201 & 83 & 118 & 0.70 \\
MS with MR & 38 & 7 & 31 & 0.22 \\
\hline Aortic Valve & 43 & 31 & 12 & 2.55 \\
AS & 5 & 5 & 0 & 5 \\
AR & 33 & 21 & 12 & 1.75 \\
AS with AR & 5 & 5 & 0 & 5 \\
Mixed involvement & 92 & 42 & 50 & 0.84 \\
AR with MR & 63 & 30 & 33 & 0.90 \\
AR with MS & 13 & 5 & 8 & 0.62 \\
AS with MR & 7 & 4 & 3 & 1.33 \\
AS with MS & 3 & 2 & 1 & 2 \\
AR/AS/MR/MS & 2 & 1 & 1 & 1 \\
AR/MS/MR & 4 & 0 & 4 &
\end{tabular}




\section{Discussion}

In our study RHD is more common in female compared to male which are similar to other studies from the other developing countries, ${ }^{7,8,9}$ although a study in a western country showed both sexes are equally affected by RHD. ${ }^{10}$

In our study most of the patients were taking Penicillin for RHD (88.4\%) compared to for RF (11.6\%) which is similar to the study done by Humagain et al. ${ }^{11}$ The penicillin injection is provided free for the patient, compliance with the treatment is good.

Our study revealed that the Mitral valve is most commonly involved than the aortic valve which is similar to other studies. ${ }^{10,12,13}$ In our study MS is the most common lesion followed by MR this finding is similar to the results in others studies, ${ }^{14,15,16}$ but a study done by Humagin ${ }^{11}$ et al and Shrestha ${ }^{17}$ et al showed $\mathrm{MR}$ as the common valvular involvement.

In our study AS was the least common similar to other studies. ${ }^{9,16,18}$ Our study revealed that mitral valve disease was more common in female than in male whereas aortic valve disease was more common in male than female. We found AS to be more common among males like the study Humagain ${ }^{11}$ et al. however Shrestha $\mathrm{N}^{17}$ et al found AR to be more common among males .

Though adverse events in patients taking Injection Benzathine Penicillin are reported in other study, during our study period we didn't had any major adverse events.

Single centre study without the detail echocardiografic measurements and clinical evaluation are the limitation of this study.

\section{Conclusions}

Our study suggests that women are more commonly affected than male. Mitral valve is the most common valve involved in RHD.

\section{Acknowledgement}

We would like to thank all the nursing staff of our Out patients department for their help in collecting the data needed for this study.

\section{References}

1 Ali SK, Eldaim IN, Osman SH, Bakhite SM. Clinical and echocardiographic features of children with rheumatic heart disease and their serum cytokine profile. The Pan African medical journal.2012;13:36.

2 Bahadur KC, Sharma D, Shrestha MP, et al. Prevalence of rheumatic and congenital heart disease in schoolchildren of Kathmandu valley in Nepal. Indian heart journal 2003;55(6):615-8.
3 Shrestha UK, Bhattarai TN, Pandey MR. Prevalence of rheumatic fever and rheumatic heart disease in school children in a rural community of the hill region of Nepal. Indian heart journal 1991;43(1):39-41.

4 Marijon E, Mirabel M, Celermajer DS,et al. Rheumatic heart disease. Lancet 2012;379(9819):953-64.

5 Bajoria D, Menon T. The HLA Class II Associations with Rheumatic Heart Disease in South Indian Patients: A Preliminary Study. Journal of clinical and diagnostic research:JCDR 2013;7(2):302-4.

6 Bo Reményi, Nigel Wilson, Andrew Steer, et al. World Heart Federation criteria for echocardiographic diagnosis of rheumatic heart disease-an evidence-based guideline. Nat. Rev. Cardiol 2012;9:297-309.

7 Marcus R. H, Sareli P, Pocock W. A,et al. The spectrum of severe rheumatic mitral valve disease in a developing country. Ann Intern Med 1994;120:177-183.

8 S. S. Danbauchi, M. A. Alhassan, S. O. David,et al. Spectrum of rheumatic heart disease in Zaria,Northen Nigeria. Annals of African Medicine 2004;3(1):17-21.

9 Khan RF, Imtiaz Y, Ali H, et al. Natural history and relative distribution of different valvular heart diseases in Mayo hospital, Lahore. Ann K E Med Coll 2002; 8: 90-1.

10 Dajani AS. Rheumatic ferver. In: Braunwald E, Ziper DP, Libby P, editors. Heart disease, a textbook of cardiovascular medicine. 6th ed, Philadelphia: WB Saunders 2001; 2192.

11 Humagain S, Gurung R, Pant $\mathrm{P}$ et al.Pattern of valvular involvement and demographic features of patients on injection Benzathine Penicillin at Dhulikhel Hospital. Nepalese Heart Journal. 2012;9(1):16-19.

12 Danbauchi SS, Alhassan MA, Wammanda R. Spectrum of rheumatic heart disease in Zaria,Northern Nigeria. Ann Afr. Med 2004;3:17-21.

13 Khan MH, Tasneem H, Khan SZ, et al. Echocardiogram:Screening for valvular heart disease in an Out-patient clinic. Med Today 2005;3(3):133-6.

14 Mclaren M. J, Hawkins D. M, Koomof H. J, et al. Epidemiology of rheumatic heart disease in black school children in Soweto. Br Med J 1975; 3:474-478.

15 Sorour A. H, El Kholy A. M. Prevalence of rheumatic heart disease in school children. J Egypt Med Assoc 1969;52:1-6.

16 Mohammad Faheem, Mohammad Hafizullah, Adnan Gul,Hikmatullah Jan,et al. Pattern of valvular lesions in rheumatic heart disease. JPMI 2007; 21(2):99-103.

17 Shrestha NR, Pilgrim T, Karki P, et al.Rheumatic heart disease revisited: patterns of valvular involvement from a consecutive cohort in eastern Nepal. J Cardiovasc Med 2012; 13(11):755-9.

18 Naim M. Rheumatic fever and RHD at the department of child health, school of medicine, University of North Sumatera. Paediatrica Indonesiana 1989;29:64-71.
Cite this article as: Rabi Malla, Suman Thapaliya, Prakash Gurung, et al. Patterns of Valvular Involvement in Rheumatic Heart Disease patients taking Benzathine Peniciliin at Shahid Gangalal National Heart Centre, Kathmandu, Nepal. Nepalese Heart Journal 2016;13(2): 25-27. 